\title{
Púrpura trombocitopênica idiopática: uma doença subdiagnosticada
}

\author{
Ana Klara Rodrigues Alves \\ Acadêmica de Enfermagem da Universidade Estadual do Piauí (UESPI) \\ \klaraphb@outlook.com \\ Barbara Beatriz Lira da Silva \\ Acadêmica de Enfermagem da Universidade Estadual do Piauí (UESPI) \\ Taynara Lais Silva \\ Acadêmica de Enfermagem da Universidade Estadual do Piauí (UESPI) \\ Ludmilla Karen Brandão Lima de Matos \\ Acadêmica de Enfermagem da Universidade Estadual do Piauí (UESPI) \\ Gustavo Wilson de Sousa Mello \\ Professor Doutor Efetivo do curso de Enfermagem da Universidade Estadual do Piauí (UESPI)
}

Recebido em 26 de setembro de 2020

Aceito em 18 de maio de 2021

\begin{abstract}
Resumo:
A púrpura trombocitopênica idiopática (PTI) é caracterizada pela destruição de plaquetas e inibição da sua produção, que se evidencia por trombocitopenia isolada. Trata-se de uma doença autoimune subdiagnosticada, pois não existe um exame próprio para diagnostica-la e devido tal circunstância a PTI é confundida com outras doenças que causam diminuição de plaquetas. Esta revisão objetiva retratar as características da púrpura trombocitopênica idiopática, como é feito seu diagnóstico e o seu tratamento. Para a construção desta revisão integrativa, realizou-se uma busca eletrônica de artigos de periodicidade, na Biblioteca Virtual de Saúde (BVS), nas bases de dados do Lilacs, Medline e Scielo (Scientific Electronic Library Online). Foram utilizados artigos escritos nas línguas portuguesa, inglesa e espanhola; publicados e indexados entre os anos 2000 e 2019 nas bases de dados escolhidas, que respondiam um dos seguintes questionamentos: quais os métodos utilizados para o diagnóstico de PTI e como realizar o tratamento nas diferentes etapas da doença? A patogênese da PTI não é completamente esclarecida, sendo relacionada a uma destruição de plaquetas e inibição da sua produção. $O$ tratamento pode ser realizado das seguintes formas: tratamento inicial, tratamento de segunda linha, tratamento da doença refratária e tratamento em situações de emergência. A PTI tratase de uma doença autoimune subdiagnosticada, pois não existe um exame próprio para diagnosticar essa patologia, devido tal circunstância a PTI é confundida com outras doenças que causam diminuição de plaquetas.

Palavras-chave: Púrpura Trombocitopênica Idiopática, Hemorragia, Trombocitopenia.
\end{abstract}

\section{Idiophatic thrombocytopenic purpura: a subdiagnosticated disease}

\begin{abstract}
:
Idiopathic thrombocytopenic purpura (ITP) is characterized by platelets destruction and inhibition of its production, which is evidenced by thrombocytopenia isolated. It is an underdiagnosed autoimmune disease, as there is no proper test to diagnose it and due to such a condition ITP is confused with other diseases that cause platelet reduction. This review aims to portray the
\end{abstract}


characteristics of idiopathic thrombocytopenic purpura, how its diagnosis is made and its treatment. For the construction of this integrative review, it was performed an electronic database search in Virtual Health Library (VHL) in the databases of the Lilacs, Medline and Scielo (Scientific Electronic Library Online). Articles written in the Portuguese, English and Spanish languages were selected, published and indexed between the years 2000 and 2019 in the chosen databases, that answered one of the following questions: what are the methods used for the diagnosis of ITP and how to perform the treatment in different stages of the disease? The PTI pathogenesis is not completely clarified, being related to a platelet destruction and inhibition of its production. Treatment can be carried out in the following ways: initial treatment, second-line treatment, treatment of refractory disease and treatment in emergency situations PTI is an autoimmune disease underdiagnosed, as there is no proper examination to diagnose this pathology, due to this circumstance the PTI is confounded with other diseases that cause platelet reduction.

Keywords: Idiophatic thrombocytopenic purpura, Bleeding, Thrombocytopenia.

\section{Púrpura trombocitopénica idiopática: una enfermedad subdiagnosticada}

\section{Resumen:}

La púrpura trombocitopénica idiopática (PTI) se caracteriza por la destrucción plaquetaria y la inhibición de su producción, que se evidencia solo con trombocitopenia. Es una enfermedad autoinmune subdiagnosticada, porque no hay una prueba adecuada para diagnosticarla y debido a tal circunstancia la PTI se confunde con otras enfermedades que causan disminución plaquetaria. Esta revisión tiene como objetivo retratar las características de la púrpura trombocitopénica idiopática, cómo se hace su diagnóstico y su tratamiento. Para la construcción de esta revisión integradora, se realizó una búsqueda electrónica de artículos de periodicidad en la Biblioteca Virtual en Salud (BVS), en las bases de datos de Lilas, Medline y Scielo (Scientific Electronic Library Online). Se utilizaron los artículos escritos en portugués, inglés y español; publicados e indexados entre 2000 y 2019 en las bases de datos elegidas, que respondieron a una de las siguientes preguntas: ¿Cuáles son los métodos utilizados para el diagnóstico de PTI y cómo realizar el tratamiento en los diferentes estadios de la enfermedad? La patogénesis de la PTI no está totalmente, aclarada estando relacionada con la destrucción de las plaquetas y la inhibición de su producción. El tratamiento se puede realizar de las siguientes maneras: tratamiento inicial, tratamiento de segunda línea, tratamiento de la enfermedad refractaria y tratamiento en situaciones de emergencia. La PTI es una enfermedad autoinmune subdiagnosticada, porque no hay un examen adecuado para diagnosticar esta enfermedad, debido a esta circunstancia la PTI se confunde con otras enfermedades que causan reducción plaquetaria.

Palabras clave: Púrpura trombocitopénica idiopática, Hemorragia, Trombocitopenia.

\section{INTRODUÇÃO}

A púrpura trombocitopênica idiopática (PTI), também conhecida como púrpura trombocitopênica imunonológica, autoimune ou isoimune, é uma doença autoimune que consiste na minimização de plaquetas, que são as células responsáveis pelo processo de coagulação do sangue, a partir da sua destruição no sistema retículo endotelial do baço (QU et al., 2018). Dessa forma, as plaquetas tornam-se desconhecidas, acarretando sua diminuição em massa. A PTI é uma doença hematológica frequente, definida por trombocitopenia isolada, podendo ser aguda, mais frequente em crianças, ou crônica, quando persiste por mais 
de 12 meses, típica do adulto (MARQUES et al., 2005). Possui este nome, púrpura, devido a um fenótipo da doença que é a presença de manchas roxas ou avermelhadas que aparecem na pele. A palavra trombocitopênica justifica-se pela diminuição do número de plaquetas e o termo idiopática devido ao desconhecimento de sua etiologia (OLIVEIRA, 1988).

A PTI acomete tanto crianças como adultos, podendo ser classificada de acordo com a faixa etária em que se manifesta e quanto ao tempo de evolução. É uma das causas mais comuns de plaquetopenia em crianças, com uma incidência anual em torno de 3 a 8 casos por 100.000 crianças, com maior número de casos entre os 2 a 5 anos de idade e com leve predomínio no sexo masculino (KURTZBERG et al., 1992). Dados de estudos epidemiológicos internacionais em adultos, que fornecem uma estimativa de incidência de 1,6-2,7 casos por 100.000 pessoas/ano e uma prevalência de 9,5-23,6 casos por 100.000 pessoas, com predominância no sexo feminino (ABRAHAMSON et al., 2009), havendo um leve discordância com o estudo de Kurtzberg (1992). No estudo de Goubran (2018) a incidência deste distúrbio é de cerca de 2 e 12/100.000 por ano para adultos e crianças, respectivamente, com mortalidade potencial em alguns casos. A PTI aguda, ocorrendo principalmente em crianças, tem um início e resolução em menos de 6 meses.

Como a maioria das doenças autoimunes, a PTI apresenta etiologia desconhecida, mas sabe-se que é provocada por anticorpos autorreativos, que se fixam nos receptores da membrana das plaquetas provocando a diminuição do tempo de vida e sobrevivência destes fragmentos de célula. Outras causas possíveis são a ruptura de capilares, sendo essa forma chamada de fragilidade capilar, que pode ser de origem alérgica, hormonal ou genética, e também pode ocorrer por carência de vitamina C no organismo (DELGADO et al., 2009; OLIVEIRA, 1988).

\section{METODOLOGIA}

Trata-se de uma revisão integrativa da literatura, que conforme SAMPAIO e MANCINI (2007) é a construção de uma análise ampla da literatura com passos pré-definidos; é um tipo de investigação disponibiliza um resumo das evidências relacionadas a uma estratégia de intervenção específica, mediante a aplicação de métodos explícitos e sistematizados de busca, 
apreciação crítica e síntese da informação selecionada, onde será possível integrar as informações de um conjunto de estudos, que podem apresentar resultados conflitantes e/ou coincidentes, bem como identificar temas que necessitam de evidência, auxiliando na orientação para investigações futuras, realizada através da Biblioteca Virtual de Saúde (BVS) nas bases de dados do Lilacs, Medline e Scielo, buscando responder o seguinte questionamento: quais os métodos utilizados para o diagnóstico de PTI e como realizar o tratamento nas diferentes etapas da doença? Por meio do cruzamento simultâneo entre os descritores "Púrpura Trombocitopênica idiopática", "Trombocitopenia" e "Hemorragia" foram encontrados 50 artigos, dos quais 33 (Quadro 1) foram selecionados. Quanto aos critérios de inclusão, introduziram-se artigos escritos na língua portuguesa e inglesa publicados entre 2000 e 2019. Em relação aos critérios de exclusão foram dispensados artigos que não fossem voltados para a temática central.

Quadro 1 - Distribuição dos artigos utilizados nesta revisão segundo o nome da publicação, autores, revista e ano.

\begin{tabular}{|c|c|c|c|}
\hline ARTIGO & AUTORES & REVISTA & $\begin{array}{c}\text { ANO DE } \\
\text { PUBLICAÇÃO }\end{array}$ \\
\hline $\begin{array}{l}\text { Therapeutic effects of } \\
\text { rituximab combined } \\
\text { with } \\
\text { cyclophosphamide on } \\
\text { refractory idiopathic } \\
\text { thrombocytopenic } \\
\text { purpura }\end{array}$ & $\begin{array}{l}\text { Wang, J.; Wang, B.; } \\
\text { Sun, Z.; Xue, K. }\end{array}$ & $\begin{array}{l}\text { Experimental and } \\
\text { Therapeutic } \\
\text { Medicine }\end{array}$ & 2019 \\
\hline $\begin{array}{l}\text { A Case of Multiple } \\
\text { Cerebral Infarction } \\
\text { Preceding Acute } \\
\text { Exacerbation of } \\
\text { Idiopathic } \\
\text { Thrombocytopenic } \\
\text { Purpura }\end{array}$ & $\begin{array}{l}\text { Sasaki, T., Yasuda, } \\
\text { T., ABE D., Myiano } \\
\text { R., Kainaga M., } \\
\text { Tomura N., } \\
\text { Kitamura M., } \\
\text { Nakayama T. e } \\
\text { Imafuku I.; }\end{array}$ & $\begin{array}{l}\text { Journal of Stroke } \\
\text { and } \\
\text { Cerebrovascular } \\
\text { Diseases }\end{array}$ & 2019 \\
\hline $\begin{array}{l}\text { Rescue therapy for } \\
\text { acute idiopathic } \\
\text { thrombocytopenic } \\
\text { purpura unresponsive }\end{array}$ & $\begin{array}{l}\text { Reynolds S.D., } \\
\text { Hashmi H., Ngo P. } \\
\text { e Kloecker G. }\end{array}$ & BMJ Case Reports & 2019 \\
\hline
\end{tabular}




\begin{tabular}{|c|c|c|c|}
\hline $\begin{array}{l}\text { to } \\
\text { conventional treatment }\end{array}$ & & & \\
\hline $\begin{array}{l}\text { A characteristic flow } \\
\text { cytometric pattern } \\
\text { with broad forward } \\
\text { scatter and narrowed } \\
\text { side scatter helps } \\
\text { diagnose immune } \\
\text { thrombocytopenia } \\
\text { (ITP) }\end{array}$ & $\begin{array}{l}\text { Araki, R., } \\
\text { Nishimura, R., } \\
\text { Kuroda, R., Fujiki } \\
\text { T., Mase S., } \\
\text { Noguche K., Ikawa } \\
\text { Y., Maeba H., } \\
\text { Yachie A. }\end{array}$ & $\begin{array}{l}\text { International } \\
\text { Journal of } \\
\text { Hematology }\end{array}$ & 2018 \\
\hline $\begin{array}{l}\text { Mean platelet volume } \\
\text { at baseline and immune } \\
\text { thrombocytopenia } \\
\text { relapse in Chinese } \\
\text { newly diagnosed } \\
\text { patients: a } \\
\text { retrospective cohort } \\
\text { study }\end{array}$ & $\begin{array}{l}\text { Chen C., Song J., } \\
\text { Wang Q., Wang } \\
\text { L.H., Guo P.X. }\end{array}$ & Hematology & 2018 \\
\hline $\begin{array}{l}\text { Flow cytometry and } \\
\text { immune } \\
\text { thrombocytopenic } \\
\text { purpura }\end{array}$ & $\begin{array}{l}\text { Goubran, H., Hart, } \\
\text { C., Othman, I., \& } \\
\text { Seghatchian, J }\end{array}$ & $\begin{array}{l}\text { Transfusion and } \\
\text { Apheresis Science }\end{array}$ & 2018 \\
\hline $\begin{array}{l}\text { Chinese guidelines for } \\
\text { treatment of adult } \\
\text { primary immune } \\
\text { thrombocytopenia. }\end{array}$ & $\begin{array}{l}\text { Liu XG, Bai XC, } \\
\text { Chen FP, et al. }\end{array}$ & $\begin{array}{l}\text { International } \\
\text { Journal of } \\
\text { Hematology }\end{array}$ & 2018 \\
\hline $\begin{array}{l}\text { Low platelet count as } \\
\text { risk factor for } \\
\text { infections in patients } \\
\text { with primary immune } \\
\text { thrombocytopenia: a } \\
\text { retrospective } \\
\text { evaluation }\end{array}$ & $\begin{array}{l}\text { Qu, M., Liu, Q., } \\
\text { Zhao, H. G., Peng, } \\
\text { J., Ni, H., Hou, M., } \\
\text { \& Jansen, A. }\end{array}$ & $\begin{array}{l}\text { Annals of } \\
\text { hematology }\end{array}$ & 2018 \\
\hline $\begin{array}{l}\text { Idiopathic } \\
\text { Thrombocytopenic } \\
\text { Purpura in Individuals } \\
\text { Older than } 75 \text { Years: A } \\
\text { Rare Series }\end{array}$ & Zulfiqar AA. & $\begin{array}{l}\text { Southern Medical } \\
\text { Journal }\end{array}$ & 2017 \\
\hline
\end{tabular}




\begin{tabular}{|c|c|c|c|}
\hline $\begin{array}{l}\text { Púrpura } \\
\text { trombocitopênica } \\
\text { idiopática }\end{array}$ & $\begin{array}{l}\text { Augusto, K. M. M., } \\
\text { Sá, J. L., Braz, B. M., } \\
\text { Barbosa, T. A., } \\
\text { Pascarelli, B. M. O. }\end{array}$ & Corpus Sci & 2015 \\
\hline $\begin{array}{l}\text { Dental considerations } \\
\text { on the management of } \\
\text { Idiopathic } \\
\text { Thrombocytopenic } \\
\text { Purpura in children: } \\
\text { case report }\end{array}$ & $\begin{array}{l}\text { Rossier, V. F., } \\
\text { Vieira, S. M. C. P. A. } \\
\text { C., Ciamponi A. L. } \\
\text { et al. }\end{array}$ & $\begin{array}{l}\text { Revista Gaúcha de } \\
\text { Odontologia }\end{array}$ & 2015 \\
\hline $\begin{array}{l}\text { Purpura } \\
\text { trombocitopenica } \\
\text { idiopática durante a } \\
\text { gravidez: relato de } \\
\text { caso. }\end{array}$ & $\begin{array}{l}\text { Vitor, R.R.R.; } \\
\text { Schwartz, T.A.C.; } \\
\text { Franca, I. B. }\end{array}$ & Revista Uningá & 2015 \\
\hline $\begin{array}{l}\text { Evaluation of the } \\
\text { diagnostic performance } \\
\text { of platelet-derived } \\
\text { indices for the } \\
\text { differential diagnosis of } \\
\text { thrombocytopenia in } \\
\text { pediatrics }\end{array}$ & $\begin{array}{l}\text { Aponte-Barrios } \\
\text { NH, Linares- } \\
\text { Ballesteros A, } \\
\text { Sarmiento-Urbina } \\
\text { IC, Uribe-Botero } \\
\text { GI. }\end{array}$ & $\begin{array}{l}\text { Revista de la } \\
\text { Facultad de } \\
\text { Medicina }\end{array}$ & 2014 \\
\hline $\begin{array}{l}\text { Púrpura } \\
\text { trombocitopenica } \\
\text { trombotica (reporte del } \\
\text { primer caso clíinico en } \\
\text { costa rica donde se } \\
\text { demuestra la presencia } \\
\text { de inhibidores de } \\
\text { adamts }\end{array}$ & $\begin{array}{l}\text { Bonilla, C. Q., } \\
\text { González, M. A. }\end{array}$ & $\begin{array}{l}\text { Revista Medica de } \\
\text { Costa Rica Y } \\
\text { Centro America }\end{array}$ & 2014 \\
\hline $\begin{array}{l}\text { Trombocitopenia } \\
\text { autoimune em crianças: } \\
\text { revisão das } \\
\text { recomendações do } \\
\text { último consenso }\end{array}$ & $\begin{array}{l}\text { Santana, L. M.; } \\
\text { Neves, T.; FenilliI, } \\
\text { A. C.; Borba, L. G.; } \\
\text { Kirst, D. et al. }\end{array}$ & $\begin{array}{l}\text { Boletim Científico } \\
\text { de Pediatria }\end{array}$ & 2013 \\
\hline $\begin{array}{l}\text { Manejo estomatológico } \\
\text { del paciente con } \\
\text { púrpura }\end{array}$ & $\begin{array}{l}\text { Granados, M. R. I., } \\
\text { Ángeles, E. T., } \\
\text { Aguirre, A. H. }\end{array}$ & $\begin{array}{l}\text { Revista } \\
\text { Odontológica } \\
\text { Mexicana }\end{array}$ & 2012 \\
\hline
\end{tabular}




\begin{tabular}{|c|c|c|c|}
\hline $\begin{array}{l}\text { trombocitopénica } \\
\text { idiopática (PTI): } \\
\text { Reporte de un caso }\end{array}$ & & & \\
\hline $\begin{array}{l}\text { Uso do eltrombopag } \\
\text { para tratamento da } \\
\text { púrpura } \\
\text { trombocitopênica } \\
\text { imune }\end{array}$ & $\begin{array}{l}\text { Savignon, L. F; } \\
\text { Matheus, M. E }\end{array}$ & $\begin{array}{l}\text { Revista Brasileira } \\
\text { de Farmácia }\end{array}$ & 2012 \\
\hline $\begin{array}{l}\text { Detección de } \\
\text { autoanticuerpos } \\
\text { antiplaquetarios por } \\
\text { ELISA en pacientes con } \\
\text { púrpura } \\
\text { trombocitopénica } \\
\text { autoinmune }\end{array}$ & $\begin{array}{l}\text { Cruz, O. Y. P, } \\
\text { Hernández, A. A. } \\
\text { B., Ravine, B. P., } \\
\text { Franch, N. F. }\end{array}$ & $\begin{array}{l}\text { Revista Archivo } \\
\text { Médico de } \\
\text { Camaguey }\end{array}$ & 2011 \\
\hline $\begin{array}{l}\text { Púrpura } \\
\text { trombocitopênica } \\
\text { idiopática: } \\
\text { etiopatogênese, } \\
\text { diagnóstico e } \\
\text { tratamento em adultos }\end{array}$ & $\begin{array}{l}\text { Krettli, W. S. C., } \\
\text { Mota, B. C., } \\
\text { Andrade, G. H. A. et } \\
\text { al. }\end{array}$ & $\begin{array}{l}\text { Revista Médica de } \\
\text { Minas Gerais }\end{array}$ & 2011 \\
\hline $\begin{array}{l}\text { The incidence of } \\
\text { idiopathic } \\
\text { thrombocytopenic } \\
\text { purpura among adults: } \\
\text { a population-based } \\
\text { study and literature } \\
\text { review }\end{array}$ & $\begin{array}{l}\text { Abrahamson, P. E., } \\
\text { Hall, S. A., Feudjo- } \\
\text { Tepie, M., Mitrani- } \\
\text { Gold F. S., Logie, J. }\end{array}$ & $\begin{array}{l}\text { European Journal } \\
\text { of Haematology }\end{array}$ & 2009 \\
\hline $\begin{array}{l}\text { Idiopathic } \\
\text { thrombocytopenic } \\
\text { purpura in childhood: a } \\
\text { population-based study } \\
\text { in Qatar }\end{array}$ & $\begin{array}{l}\text { Al-Mulla, N., } \\
\text { Bener, A., Amer, A., } \\
\text { Laban, M. A }\end{array}$ & Jornal de Pediatria & 2009 \\
\hline $\begin{array}{l}\text { Primary immune } \\
\text { thrombocytopenia: } \\
\text { understanding } \\
\text { pathogenesis is the key } \\
\text { to better treatments }\end{array}$ & Chong, B. H. & $\begin{array}{l}\text { Journal of } \\
\text { Thrombosis and } \\
\text { Haemostasis }\end{array}$ & 2009 \\
\hline
\end{tabular}




\begin{tabular}{|c|c|c|c|}
\hline $\begin{array}{l}\text { Púrpura } \\
\text { trombocitopênica } \\
\text { imune da criança: } \\
\text { experiência de } 12 \text { anos } \\
\text { em uma única } \\
\text { instituição brasileira }\end{array}$ & $\begin{array}{l}\text { Delgado, R. B., } \\
\text { Viana, M. B., } \\
\text { Fernandes, R. A. F. }\end{array}$ & $\begin{array}{l}\text { Revista Brasileira } \\
\text { de Hematologia e } \\
\text { Hemoterapia }\end{array}$ & 2009 \\
\hline $\begin{array}{l}\text { Characteristics of } \\
\text { immune } \\
\text { thrombocytopenic } \\
\text { purpura: a guide for } \\
\text { clinical practice }\end{array}$ & Provan, D. & $\begin{array}{l}\text { European Journal } \\
\text { of Haematology } \\
\text { Supplementum }\end{array}$ & 2009 \\
\hline $\begin{array}{l}\text { Immune } \\
\text { thrombocytopenic } \\
\text { purpura in adults }\end{array}$ & $\begin{array}{l}\text { Godeau, B., Provan, } \\
\text { D., Busse, J. }\end{array}$ & $\begin{array}{l}\text { Current Opinion in } \\
\text { Hematology }\end{array}$ & 2007 \\
\hline $\begin{array}{l}\text { Púrpura } \\
\text { trombocitopênica } \\
\text { idiopática e linfoma } \\
\text { não-Hodgkin de células } \\
\text { T na infância }\end{array}$ & $\begin{array}{l}\text { Borges, A. C., Pizza, } \\
\text { M., Borsato, M. L., } \\
\text { Silva, H. R. M., } \\
\text { Castro, H. C. et al. }\end{array}$ & $\begin{array}{l}\text { Revista Brasileira } \\
\text { de Hematologia e } \\
\text { Hemoterapia }\end{array}$ & 2006 \\
\hline $\begin{array}{l}\text { Púrpura } \\
\text { Trombocitopênica } \\
\text { Idiopática (PTI): relato } \\
\text { de caso clínico em } \\
\text { paciente jovem no } \\
\text { município de Maringá- } \\
\text { PR. }\end{array}$ & $\begin{array}{l}\text { Cardoso, R. C. S., } \\
\text { Posso, K. F., } \\
\text { Henrique, T. M. }\end{array}$ & Revista Uningá & 2005 \\
\hline $\begin{array}{l}\text { Angioplastia } \\
\text { Transluminal } \\
\text { Coronariana em } \\
\text { Portador de Púrpura } \\
\text { Trombocitopênica } \\
\text { Idiopática }\end{array}$ & $\begin{array}{l}\text { Marques, L.G.M.; } \\
\text { Furukawa, M. K.T; } \\
\text { Leitão, H. P., } \\
\text { Quiñones, J.L. A., et } \\
\text { al. }\end{array}$ & $\begin{array}{l}\text { Arquivos } \\
\text { Brasileiros de } \\
\text { Cardiologia }\end{array}$ & 2005 \\
\hline $\begin{array}{l}\text { Esplenectomia vídeo- } \\
\text { laparoscópica para } \\
\text { púrpura } \\
\text { trombocitopênica } \\
\text { imune: técnica e } \\
\text { resultados }\end{array}$ & $\begin{array}{l}\text { Zorrón, R.; Neto, S. } \\
\text { H. C.; Kanaan, E.; } \\
\text { Toaspern, T. V.et } \\
\text { al. }\end{array}$ & $\begin{array}{l}\text { Revista do Colégio } \\
\text { Brasileiro de } \\
\text { Cirurgiões }\end{array}$ & 2004 \\
\hline
\end{tabular}




\begin{tabular}{|l|l|l|l|}
\hline $\begin{array}{l}\text { Initial management of } \\
\text { immune } \\
\text { thrombocytopenic } \\
\text { purpura in adults: a } \\
\text { randomized controlled } \\
\text { trial comparing } \\
\text { intermittent anti-D } \\
\text { with routine care. }\end{array}$ & $\begin{array}{l}\text { GeorgeE, J. N., } \\
\text { Raskob, G. E., } \\
\text { Mesely S. K., } \\
\text { R. M., Cobos, E. et } \\
\text { al. }\end{array}$ & $\begin{array}{l}\text { American Journal } \\
\text { of Hematology }\end{array}$ & 2003 \\
\hline $\begin{array}{l}\text { Púrpura } \\
\text { trombocitopénica } \\
\text { idiopática }\end{array}$ & $\begin{array}{l}\text { Gomar, M. H., } \\
\text { Tapia, N. C. C., } \\
\text { Cervera, J. L. }\end{array}$ & Médica Sur & \\
\hline $\begin{array}{l}\text { Púrpura } \\
\text { trombocitopênica após } \\
\text { vacina de hepatite B }\end{array}$ & $\begin{array}{l}\text { Maezono, R., } \\
\text { Escobar, A. M. U. }\end{array}$ & Jornal de Pediatria & \\
\hline $\begin{array}{l}\text { Idiopathic autoimmune } \\
\text { thrombocytopenic } \\
\text { purpura }\end{array}$ & $\begin{array}{l}\text { Kurtzberg, J., } \\
\text { Stockman, J. A., et } \\
\text { al. }\end{array}$ & $\begin{array}{l}\text { Advances in } \\
\text { Pediatrics }\end{array}$ & 2003 \\
\hline
\end{tabular}

Fonte: Dados do trabalho.

\section{RESULTADOS}

A patogênese da PTI não é completamente esclarecida, sendo relacionada a uma destruição de plaquetas e inibição da sua produção. A destruição das plaquetas ocorre pelos macrófagos presentes no baço (sistema monuclear fagocitário) e é determinada pela produção de IgG pelos linfócitos B. Múltiplos antígenos na superfície plaquetária parecem ser responsáveis pela ligação com anticorpos, especialmente a glicoproteína IIb/ IIIa e a Ib/IX, que quando ligados desencadeiam o processo de destruição de plaquetas (CHONG, 2009; GOUBRAN et al., 2018). KRETTLI (2011) demonstrou que os autoanticorpos que promovem a destruição das plaquetas estão presentes em 50 a $70 \%$ dos pacientes, o que indica outro mecanismo de destruição a ser investigado. STAHL (1986) mostrou que certos anticorpos antiplaquetários reagem apenas a megacariócitos que se encontram em estado de trombocitopoese (início da produção plaquetária). A trombopoetina endógena (TPO), 
hormônio que controla o desenvolvimento dos megacariócitos e produção das plaquetas, encontra-se aumentada em casos de plaquetopenia, porém somente discreto aumento pode ser encontrado na PTI (PROVAN, 2009).

Esta patologia não apresenta etiologia definida, podendo surgir depois de infecções virais nas quais os anticorpos antivirais promovem reação cruzada com as glicoproteínas plaquetárias, ou também, reações a medicamentos ou vacinas (QU et al., 2018; GRANADOS et al., 2012). KRETTLI (2011) ainda atenta que alterações e anormalidades na resposta imune podem levar à produção de autoanticorpos e a outras síndromes autoimunes que apresentam trombocitopenia. A PTI às vezes é acompanhada por um aumento de megacariócitos da medula óssea e sua incidência é relativamente alta em crianças (WANG et al., 2019).

A púrpura trobocitopênica idiopática pode ser completamente assintomática e ter a trombocitopenia como única indicação. Nesse caso, o diagnóstico pode ser realizado primeiramente através da história clínica, do exame físico e do hemograma completo para verificar se há redução no número de plaquetas. Outro método diagnóstico é o esfregaço de sangue periférico. $\mathrm{O}$ diagnóstico é confirmado por exclusão de outras causas que levam a redução no número de plaquetas, como leucemia, linfomas, doenças infecciosas, HIV, neoplasias, hepatopatias, doenças genéticas e outras patologias autoimunes, devendo ser feita a avaliação da medula (biópsia e aspirado) para confirmar ou excluir a doença. Não há exames com especificidade e sensibilidade suficiente para confirmar a PTI (MAEZONO et al., 2000; BRASIL, 2013; CRUZ et al., 2011; AL-MULLA et al., 2009; APONTE-BARRIOS et al., 2014; SANTANA et al., 2013). No entanto, ARAKI (2018) demonstrou que a avaliação do tamanho das plaquetas por citometria de fluxo foi útil no diagnóstico de trombocitopenia e identificaram os parâmetros associados com manifestações de sangramento na mucosa ou órgão. GOUBRAN (2018) confirmou que a citometria de fluxo pode ser usada como uma ferramenta de diagnóstico auxiliar. A citometria também é amplamente utilizada como uma ferramenta para melhor compreensão da etiologia imune da doença e pode ajudar a identificar o prognóstico de marcadores. A doença é considerada persistente quando houver plaquetopenia nos 3 a 12 meses após o diagnóstico e crônica quando persistir por mais de 12 meses (BONILLA et al., 2014; ROSSIER et al., 2015, BORGES et al., 2006). 


\section{DISCUSSÃO}

O tratamento pode ser realizado das seguintes formas: tratamento inicial, tratamento de segunda linha, tratamento da doença refratária e tratamento em situações de emergência. No primeiro, não há necessidade de tratamento. Ocorre no caso de os pacientes estarem com a plaquetopenia leve à moderada e clínica assintomática (SAVIGNON et al., 2012). À medida que a trombocitopenia se torna grave $\left(<20.000 / \mathrm{mm}^{3}\right)$ ou diante de sangramentos, começa a ser necessário o uso da farmacoterapia. A pulsoterapia com corticoesteroides constitui a escolha para o tratamento inicial da PTI. Outros esquemas de administração também são utilizados, porém sem evidência de superioridade de um sobre o outro. Em adultos, o medicamento inicial de escolha é a aplicação de prednisona oral, $2 \mathrm{mg} / \mathrm{kg}$, em dose única diária. A duração do tratamento com a prednisona e a posologia são determinados pela contagem de plaquetas (REYNOLDS et al., 2019; AUGUSTO et al., 2015; ZAGO et al., 2013; GOMAR et al., 2003. SAVIGNON et al., 2012; ROSSIER et al., 2015).

Outra medida usada é o uso de imunoglobulina intravenosa, a qual é preparada a partir de vários plasmas de doadores com anticorpos normalmente presentes no sangue do adulto. As imunoglobulinas agem por intermédio do bloqueio de receptores Fc de células do sistema retículo-endotelial. Não há consenso sobre a sua melhor posologia. Para GODEAU (2007) não apresenta superioridade terapêutica com seu uso combinado ou não com o corticosteroide em relação à monoterapia corticosteroidea. Está indicada em casos de sangramento volumosos ou pré-procedimentos cirúrgicos. A dose usual é de $400 \mathrm{mg} / \mathrm{kg} / \mathrm{dia}$ por cinco dias ou $1 \mathrm{~g} / \mathrm{kg} /$ dia por dois dias (REYNOLDS et al., 2019; ROSSIER et al., 2015; MAEZONO et al., 2000; GOMAR et al., 2003). O uso de imunoglobulina antiD é eficaz em pacientes $\mathrm{Rh}$ positivos $(\mathrm{D}+)$, porém não possui vantagem clínica significativa quando comparado a imunoglobulina intravenosa (ZAGO et al., 2013; GEORGE et al., 2003; SANTANA et al., 2013; BRASIL, 2013; GOMAR et al., 2003; KRETTLI et al., 2011). Os principais determinantes do tempo de tratamento são a contagem de plaquetas e o esquema terapêutico utilizado (VITOR, et al, 2015). Chen (2018) para pacientes adultos com diagnóstico de PTI, a recidiva é frequente após o tratamento com terapia de primeira linha, cerca de $50 \%$ dos pacientes recaem dentro de 6 meses.

O tratamento de segunda linha é executado diante da ineficiência da corticoterapia e do uso de imunoglobulina humana. Nesses casos, a opção terapêutica é a esplenectomia, pois 
o baço é o principal responsável pela destruição plaquetária, além de conter $25 \%$ da massa linfoide envolvida na produção de anticorpos. A esplenectomia é também a opção terapêutica na PTI aguda refratária e na forma crônica com necessidade de corticoterapia crônica. A contagem de plaquetas deve ser acima de $50.000 / \mathrm{mm}^{3}$ para a realização da esplenectomia e os riscos da cirurgia devem ser analisados antes da sua indicação (ZORRÓN et al., 2004; KRETTLI et al., 2011; AUGUSTO et al., 2015; CARDOSO et al., 2005). Todavia para Zago (2013) essa modalidade de tratamento é a que apresenta o melhor índice de resposta favorável, ou seja, em torno de 70\% a longo prazo. O efeito da esplenectomia é imediato e está relacionado à retirada do principal local de fagocitose das plaquetas sensibilizadas, e, secundariamente, existe também redução da produção do autoanticorpo. A morbidade da cirurgia é muito baixa, menor do que 5\%, tendo sido relatados casos de abscesso subfrênico, infecção ou trombose venosa no pós-operatório.

A doença refratária é tratada inicialmente com o uso de azatioprina ou ciclofosfamida. A falha terapêutica com a administração desses agentes requer o uso de danazol, exceto em crianças e adolescentes, devido ao seu potencial de virilização e da sua segurança incerta nessa faixa etária. A vincristina é reservada aos casos de falha terapêutica ou refratariedade aos demais agentes (ZAGO et al., 2013; BRASIL, 2013). Wang (2019), atualmente, a ciclofosfamida e o rituximabe são frequentemente utilizados no tratamento de pacientes com PTI refratária. A eficácia do rituximabe associado à ciclofosfamida no tratamento de pacientes com PTI refratária é superior à terapia tradicional com um único agente.

Em casos de situações de emergência, o tratamento utilizado é a transfusão de plaquetas, indicado quando ocorrem casos de sangramentos intracranianos ou mucoso associado à instabilidade hemodinâmica ou respiratória. Essa medida é utilizada principalmente em hemorragia com risco de morte e deve ser considerada quando sua contagem de plaquetas for inferior a 5.000 ou $10.000 / \mathrm{mm}^{3}$ e diante de paciente com febre. A imunoglobulina humana intravenosa também é usada em situações emergências, uma vez que produz respostas mais rápidas do que a corticoterapia, entretanto, seu custo é bem mais elevado e mais usado diante de urgência e gravidade (KRETTLI et al., 2011; GRANADOS et al., 2012; SANTANA et al., 2013). Mas, para Araki (2018) a falta de biomarcadores confiáveis para o risco de hemorragia grave dificulta a decisão sobre a intervenção terapêutica inicial apropriada. 


\section{CONCLUSÃO}

A PTI trata-se de uma doença autoimune subdiagnosticada, pois não existe um exame próprio para diagnosticar essa patologia, devido tal circunstância a PTI é confundida com outras doenças que causam diminuição de plaquetas. O diagnóstico da PTI é baseado na história clínica do paciente e o achado de plaquetopenia sem causa definida, ou seja, a partir de testes de exclusão de doenças mais graves, como por exemplo, a leucemia. Essa doença, apesar das complicações, é benigna e para o seu tratamento é necessário a administração de medicamentos adequados, sendo os mais utilizados corticosteroides ou imunoglobulina endovenosa (IgG). A maioria dos casos de pacientes com PTI são controlados, porém quando não tratado de maneira correta pode ser fatal. Desse modo, é fundamental que os profissionais de saúde tenham conhecimento sobre a PTI, para que não ocorram casos subdiagnosticados da doença.

\section{REFERÊNCIAS}

ABRAHAMSON, P. E., HALL, S. A., FEUDJO-TEPIE, M., MITRANI-GOLD F. S., LOGIE, J. The incidence of idiopathic thrombocytopenic purpura among adults: a population-based study and literature review. Eur J Haematol. V. 83, n. 2, p. 83-9, 2009.

AL-MULlA, N., BENER, A., AMER, A., LABAN, M. A. Idiopathic thrombocytopenic purpura in childhood: a population-based study in Qatar. J Pediatr (Rio J), v. 85, n. 3, p.269-272, 2009.

ARAKI, R., NISHIMURA, R., KURODA, R., FUJIKI, T., MASE, S., NOGUCHI, K., IKAWA, Y., MAEBA, H., YACHIE, A. A characteristic flow cytometric pattern with broad forward scatter and narrowed side scatter helps diagnose immune thrombocytopenia (ITP). Int J Hematol, v. 108, p. 151, 2018.

APONTE-BARRIOS, N. H., LINARES-BALLESTEROS, A., SARMIENTO-URBINA, I.C., URIBE-BOTERO, G.I. Evaluation of the diagnostic performance of platelet-derived indices for the differential diagnosis of thrombocytopenia in pediatrics. Rev. Fac. Med., v. 62, n. 4, p. 547-552, 2014.

AUgUSTO, K. M. M., SÁ, J. L., BRAZ, B. M., BARBOSA, T. A., PASCARELLI, B. M. O. Púrpura trombocitopênica idiopática. Corpus sci., Rio de Janeiro, jul./dez., v. 11, n. 2, p. 69 - 78, 2015.

BONILLA, C. Q., GONZÁLEZ, M. A. Púrpura trombocitopenica trombotica (reporte del primer caso clíinico en costa rica donde se demuestra la presencia de inhibidores de adamts 13). REVISTA MEDICA DE COSTA RICA Y CENTROAMERICA. v. 71, n. 612, p. $723-728,2014$.

BORGES, A. C., PIZZA, M., BORSATO, M. L., SILVA, H. R. M., CASTRO, H. C., LUPORINI, S. M., BRUNIERA P. Púrpura trombocitopênica idiopática e linfoma não-Hodgkin de células T na infância. Rev. bras. hematol. hemoter., v. 28 , n. 1, p. 73-75, 2006.

BRASIL. Ministério da Saúde. Protocolo clínico e diretrizes terapêuticas: púrpura trombocitopênica idiopática. Brasília, 2013. 
CARDOSO, R. C. S., POSSO, K. F., HENRIQUE, T. M. Púrpura Trombocitopênica Idiopática (PTI): relato de caso clínico em paciente jovem no município de Maringá-PR. Revista UNINGÁ, n. 4, p. 139-144, abr./jun. 2005.

CHEN C., SONG, J., WANG, Q., WANG, L., GUO, P. Mean platelet volume at baseline and immune thrombocytopenia relapse in Chinese newly diagnosed patients: a retrospective cohort study, Hematology, v. 23, n. 9, p. 643-652, 2018.

CHONG, B. H. Primary immune thrombocytopenia: understanding pathogenesis is the key to better treatments. J Thromb Haemost, v. 7, p. 319-21, 2009.

CRUZ, O. Y. P, HERNÁNDEZ, A. A. B., RAVINE, B. P., FRANCH, N. F. Detección de autoanticuerpos antiplaquetarios por ELISA en pacientes con púrpura trombocitopénica autoinmune. Arch. méd. Camaguey., v. 5, n. 4, p. 653664, 2011.

DELGADO, R. B., VIANA, M. B., FERNANDES, R. A. F. Púrpura trombocitopênica imune da criança: experiência de 12 anos em uma única instituição brasileira. Revista Brasileira de Hematologia e Hemoterapia, São Paulo, v. 31, n. 1, p. 29-36, 2009.

GEORGE, J. N., RASKOB, G. E., VESELY, S. K., MOORE, D., LYONS, R. M., COBOS, E., TOWELL, B. L., KLUG, P., GUTHRIE, T. H. Initial management of immune thrombocytopenic purpura in adults: a randomized controlled trial comparing intermittent anti-D with routine care. Am J Hematol., v. 74, n. 3, p. 161-169, 2003.

GODEAU, B., PROVAN, D., BUSSE, J. Immune thrombocytopenic purpura in adults. Curr Opin Hematol., v. 14, p. 535-56, 2007.

GOMAR, M. H., TAPIA, N. C. C., CERVERA, J. L. Púrpura trombocitopénica idiopática. Médica Sur, México. 2003, v.10, n.2.

GOUBRAN, H. HARTA, C., OTHMAN, B., SEGHATCHIAN, J. Flow cytometry and immune thrombocytopenic purpura. Transfusion and Apheresis Science. v. 57, n. 6, p. 800-803, Dezembro de 2018.

GRANADOS, M. R. I., ÁNGELES, E. T., AGUIRRE, A. H. Manejo estomatológico del paciente con púrpura trombocitopénica idiopática (PTI). Reporte de un caso, Revista Odontológica Mexicana. 2012, v.16, n.1, p.5357.

LIU, X. G., BAI, X. C., CHEN, F. P. ,CHENG ,Y., DAI. K., FANG, M., FENG, J., GONG, Y., GUO, T., GUO, X., HAN, Y., HONG, L., HU, Y., HUA, B., HUANG, R., LI, Y., PENG, J., SHU, M., SUN, J., SUN, P., SUN, Y., WANG, C., WANG, S., WANG, X., WU, C., WU, W., YAN, Z., YANG, F., YANG, L., YANG, R., YANG, T., YE, X., ZHANG, G., ZHANG, L., ZHENG, C., ZHOU, H., ZHOU, M., ZHOU, R., ZHOU, Z., ZHU, H., ZHU, T., HOU, M. Chinese guidelines for treatment of adult primary immune thrombocytopenia. Int J Hematol., v.107,n.6, p.615-623, Junho- 2018

KRETTLI, W. S. C., MOTA, B. C., ANDRADE, G. H. A., GONTIJO, J. R., SILVA, J. S., LOPES, J. H. M., LAFETÁ, L., BRANDÃO, L. I., ALVES, L., SILVA, R. P. Púrpura trombocitopênica idiopática: etiopatogênese, diagnóstico e tratamento em adultos. Rev Med Minas Gerais, v.21, n.4, S1-S143, 2011.

KURTZBERG, J., STOCKMAN, J. A. Idiopathic autoimmune thrombocytopenic purpura. Adv Pediatr. v.41, p.34111, 1994.

MAEZONO, R., ESCOBAR, A. M. U. Púrpura trombocitopênica após vacina de hepatite B. Jornal de Pediatria, v.76, v.5, p.395-398, Rio de Janeiro, 2000.

MARQUES, L.G.M.; FURUKAWA, M. K. T; LEITÃO, H. P., QUIÑONES, J. L. A., QUEIROZ, F. C., TIOSSI, R. F., FRANCO JR, V. R., DOMINGUES, C. E. M., SALVADORI JR, D. Angioplastia Transluminal Coronariana em Portador de Púrpura Trombocitopênica Idiopática. Arquivos Brasileiros de Cardiologia. v. 84, n. 4, p. 337-339, abril 2005.

OLIVEIRA, H. Hematologia clínica. Atheneu, 3. ed. Rio de Janeiro, 1988. 
PROVAN, D. Characteristics of immune thrombocytopenic purpura: a guide for clinical practice. Eur J Haematol Suppl. n.71, p.8-12. Mar., 2009.

QU, M., LIU, Q., ZHAO, H.G., PENG, J., NI, H., HOU, M., JANSEN, A. J. G. Low platelet count as risk factor for infections in patients with primary immune thrombocytopenia: a retrospective evaluation. Ann Hematol. Doi: https://doi.org/10.1007/s00277-018-3367-9, 2018.

REYNOLDS, S.B., HASHMI, H., NGO, P., KLOECKER, G. Rescue therapy for acute idiopathic thrombocytopenic purpura unresponsive to conventional treatment. BMJ Case Rep. doi:10.1136/bcr-2018-227717, 2019.

ROSSIER, V. F., VIEIRA, S. M. C.P. A. C., CIAMPONI, A. L., GUARÉ, R. O. Dental considerations on the management of Idiopathic Thrombocytopenic Purpura in children: case report. RGO, Rev Gaúch Odontol, Porto Alegre, v.63, n.4, p.472-476,out./dez., 2015.

SANTANA, L. M.; NEVES, T.; FENILLI, A. C.; BORBA, L. G.; KIRST, D., Fetter, F., TANIGUCHI, A. N., SABARROZ, V., MICHALOWSKI, M. B. Trombocitopenia autoimune em crianças: revisão das recomendações do último consenso. Boletim Científico de Pediatria. v.2, n.3, 2013.

SASAKI, T., YASUDA, T., ABE, D., MIYANO, R., KAINAGA, M., TOMURA, N., KITAMURA, M., NAKAYAMA, T., IMAFUKU, I. A Case of Multiple Cerebral Infarction Preceding Acute Exacerbation of Idiopathic Thrombocytopenic Purpura. Journal of Stroke and Cerebrovascular Diseases, v.28, n.3, p.789 - 791, 2019.

SAVIGNON, L. F, MATHEUS, M. E. Uso do eltrombopag para tratamento da púrpura trombocitopênica imune. Rev. Bras. Farm. v.93, n.3, p.304-309, 2012.

SAMPAIO, R.F., MANCINI, M.C. Estudos de revisão sistemática: um guia para síntese criteriosa da evidência científica. Rev. bras. fisioter., v.11, n.1, p.83-89. jan.fev. 2007.

VITOR, R.R.R., SCHWARTZ, T.A.C., FRANCA, I. B. Purpura trombocitopenica idiopática durante a gravidez: relato de caso. Revista Uningá., v.43, p. 51-54, Jan - Mar, 2015

WANG, J., WANG, B., SUN, Z., XUE, K. Therapeutic effects of rituximab combined with cyclophosphamide on refractory idiopathic thrombocytopenic purpura. Experimental and Therapeutic Medicine. v.17, n.3, p.21372142, 2019.

ZAGO, M. A., FALCÃO, R.P., PASQUINI, R. Tratado de Hematologia, In: LOURENÇO, Dayse Maria. cap. 63, p. 605611. Ed. 1. Editora Atheneu, São Paulo, 2013.

ZORRÓN, R., NETO, S. H. C., KANAAN, E., TOASPERN, T. V., CHAVES, L. P., FILHO, D. M. Esplenectomia vídeolaparoscópica para púrpura trombocitopênica imune: técnica e resultados. Rev. Col. Bras. Cir. v.31, n.4, p.265270, jul. / ago. 2004.

ZULFIGAR, A. A. Idiopathic Thrombocytopenic Purpura in Individuals Older than 75 Years: A Rare Series. South Med J. v.110, n.9,2017.

\section{(c) $)$ EY}

Este trabalho está licenciado com uma Licença Creative Commons - Atribuição 4.0 Internacional. 